Sociologie et sociétés

\title{
Réciprocités sociales. Lectures de Simmel
}

Présentation

\section{Social Reciprocities. Contemporary Simmel}

\section{Content}

\section{Gregor Fitzi et Denis Thouard}

Volume 44, numéro 2, automne 2012

Réciprocités sociales. Lectures de Simmel

Social reciprocities. Contemporary Simmel

URI : https://id.erudit.org/iderudit/1012918ar

DOI : https://doi.org/10.7202/1012918ar

Aller au sommaire du numéro

Éditeur(s)

Les Presses de l’Université de Montréal

ISSN

0038-030X (imprimé)

1492-1375 (numérique)

Découvrir la revue

Citer ce document

Fitzi, G. \& Thouard, D. (2012). Réciprocités sociales. Lectures de Simmel :

présentation. Sociologie et sociétés, 44(2), 5-18.

https://doi.org/10.7202/1012918ar 


\section{Réciprocités sociales}

Lectures de Simmel

\section{GREGOR FITZI}

Institut für Sozialwissenschaften

Soziologische Theorie

Universität Oldenburg

Ammerländer Heerstraße 114-118

D-26111 Oldenburg

Deutchsland

Courriel: gregor.fitzi@uni-oldenburg.de

\section{DENIS THOUARD}

CNRS-EHESS

CRIA-Centre de recherches interdisciplinaires

sur l'Allemagne

Centre Marc Bloch de Berlin

96, Boulevard Raspail

75006 Paris

Courriel: thouard@cmb.hu-berlin.de

$\mathrm{L}$ A RÉciprocité est sans doute le concept central de la pensée de Simmel. Un ensemble d'individus attablés à un café ou un groupe de promeneurs, des personnes prises dans un embouteillage ou dans une controverse, ne relèvent en effet d'un intérêt sociologique que lorsque l'on peut montrer que le comportement de chacun prend en compte la présence et l'action des autres. Chacun modifie imperceptiblement sa présentation de lui-même en fonction des autres. Ainsi s'établissent les formes de l'interaction sociale, des plus éphémères aux plus durables: une production et une dissolution rythmique de liens constituent le tissu social. Parfois, la réciprocité ne se produit pas, ou pas tout de suite, ou autrement qu'attendue: ce sont alors autant d'occasions de s'interroger. Se fondant sur la rencontre de plusieurs sujets, la société diffère des objets des sciences naturelles et requiert le développement d'une épistémologie spécifique. Des individus présentent un intérêt sociologique en tant qu'ils partagent une même dimension de reconnaissance mutuelle. On accorde en cela que chacun est tour à tour sujet et objet d'action réciproque, organisant son insertion dans un ensemble partagé tout en étant considéré par les autres, et intégrant leur regard dans son comportement. À la différence de Parsons, cela n'implique toutefois pas selon Simmel l'établissement d'un contexte normatif de l'action. Le maintien des formes 
sociales demande une continuité d'action réciproque fondée sur les petites ficelles du lien social quotidien qui échappent à l'attention de l'observateur concentré sur les dimensions macro-structurelles du social. Ce sont alors les catégories de la contingence spatiale et temporelle qui deviennent l'objet du regard sociologique simmelien: accélération, distance, stéréotypisation, éphémérisation du lien social. Leur étude permet d'apprécier la dynamique des sociétés complexes, en expliquant les institutions portantes, à partir de l'argent, grâce à l'analyse des formes de l'action réciproque qui en garantissent la subsistance. La réciprocité fonde l'objet de la sociologie attachée aux formes de l'association, comme elle engage la réflexion sur les limites de l'idéal d'objectivation produit par les sciences naturelles. Elle joue aussi entre le sociologue et son objet, en se retournant sur lui. Le sociologue n'est plus le roi de l'objectivation universelle, comme le voulait Durkheim, mais participe de ce qu'il étudie tout en explicitant les relations latentes qu'il entretient avec son domaine de recherche. Comment pourrait-on lui demander d'abandonner sa propre subjectivité, si elle est à ce point constitutive de la nature de son objet? La réponse est à chercher dans la fondation épistémologique de la sociologie de Simmel: la société est elle-même une «forme objective de consciences subjectives » et c'est en tant que telle qu'il faut l'étudier.

Une des conséquences de l'application simmelienne de la réciprocité à l'analyse sociologique est ainsi de faire place à un questionnement philosophique constamment ouvert des assomptions axiomatiques qui fondent la sociologie en tant que science positive. La dualité de régime des écrits simmeliens, alternant le travail sociologique et l'ambition de trouver réponse aux questionnements classiques de la philosophie audelà de ses bornes académiques, trouve ici sa raison d'être: l'un ne va pas sans l'autre. Cette dynamique épistémologique, pour fondée qu'elle soit dans les nécessités mêmes de la pensée de Simmel, entraîne les reproches d'ambiguïté et d'une insuffisante scientificité ressassés par une certaine réception encore liée à un modèle progressif lointainement hérité des stades comtiens. Elle comporte toutefois aussi des difficultés herméneutiques plus profondes, liées précisément au statut épistémologique dévolu au concept de réciprocité.

Avec l'analyse des crises et des conflits, c'est la question de savoir comment la société peut se maintenir sans céder à ses tendances autodestructrices qui passe au centre de l'attention. La leçon marxienne est très présente: la dynamique de la modernité est à chercher dans le conflit entre forces productives et rapports de production ${ }^{1}$. Elle ne caractérise toutefois pas seulement l'économie, mais tous les domaines de la culture. Mais la conception simmelienne de la modernité diffère du matérialisme historique par l'absence d'un projet de réconciliation dialectique de ses conflits. Il n'y a pas de rédemption, même à travers une révolution politique. La tâche de la sociologie consiste alors dans un diagnostic des tendances du changement social actuel sans prétendre déboucher sur une prévision objective de l'évolution de la société à venir.

1. Elle est du reste soulignée par Simmel lui-même dans le texte «Transformation des formes culturelles» ici traduit par Barbara Thériault. 
C'est ainsi que le conflit de la culture constitue selon Simmel le destin inéluctable de l'humanité moderne. La science sociale peut en décrire les émergences sans pour autant en résoudre les tensions. Cette position a de quoi décevoir plusieurs attentes. Tous les aspects normatifs de la problématique moderne y sont en effet consignés à la sphère pratique. En la matière, l'option de Simmel est l'éthique individuelle. Il n'y a pas de projet politique à réaliser parce que la complexité moderne est perçue comme un phénomène qui n'est pas réductible à la logique d'un seul système fonctionnel, mais doit être prise dans l'action réciproque de plusieurs systèmes. Ce sont les individus qui, avec le choix de leurs rôles sociaux, relient les différents domaines fonctionnels selon des combinaisons toujours nouvelles. Ainsi, ils gèrent les tensions internes à la structure sociale et affrontent le conflit de la culture moderne. En tant que science positive, la sociologie doit donc se concentrer sur l'action réciproque entre les acteurs et les différents domaines sociaux en explicitant leur dynamique. Elle est un témoin de la réalité sociale moderne, sans chercher à en réduire la complexité en termes théoriques comme le faisait la philosophie politique jusqu'à Hegel. C'est en un certain sens le dernier mot de Simmel sur la condition humaine moderne. Dans le contexte d'une réalité sociale qui présente une déliaison des hégémonies, une interconnexion croissante et une difficulté à reconstituer le sens du social dans une grande narration, cette approche contribue à rendre particulièrement actuelle la pensée de Simmel. On constatera que son intelligence des phénomènes sociaux conserve, voire reçoit une fécondité nouvelle avec l'accentuation des modernisations et des crises dont il pu repérer souvent les prémices.

\section{RÉCIPROCITÉ ET GLOBALISATION}

Par son évolution récente, le monde contemporain s'assimile de plus en plus à une «action réciproque généralisée ». Dans son principe, ce que l'on entend par la «mondialisation» n'est en effet rien d'autre qu'un dépassement de la bipartition géopolitique du globe vers une interconnexion de plusieurs centres d'influence. L'hégémonie d'une superpuissance à laquelle on s'était accoutumée, encore présente en de nombreux domaines, notamment militaro-industriel, est néanmoins efficacement contestée sur le plan économique, culturel, démographique ou des relations internationales. Un processus de décentrement s'opère, qui sape également l'idée de hiérarchie au profit de relations à double entrée, présentant des structures en réseau. Les cadres normatifs s'estompent et sont toujours plus difficiles à défendre, voire à établir. Des valeurs s'opposent sans toujours que ce soit au sein de la mutuelle reconnaissance des espaces particuliers. Le système de la finance internationale aveugle de son opacité jusqu'à ses propres acteurs. Le citoyen contemple avec un mélange d'étonnement et d'atterrement un jeu dont on lui dissimule les opérations, mais dont il pressent qu'elles n'ont rien de glorieux. Les règles du droit international, des législations nationales, des accords commerciaux constituent un entremêlement désordonné de préceptes, dont l'application dépend le plus souvent de compromis, de transactions et de rapports de force. La polycentralité s'étend à toujours plus de domaines, obérant la 
lisibilité d'un monde dont les codes sont devenus multiples. En avance sur le diagnostic actuel d'une fluidification des rapports sociaux (Baumann), Simmel a d'emblée posé la question de la consistance des formes dans un monde en changement perpétuel. Il ne propose ni une philosophie des formes symboliques (Cassirer), ni une théorie entièrement dédiée à l'élucidation de l'émergence du symbolique, à partir de l'imaginaire social (Castoriadis), ni une pensée de l'équilibre entre la violence primitive et les structures collectives qui permettent de la supporter ou de la détourner (Gehlen, Blumenberg), mais une réflexion différenciée sur l'instauration du monde de l'esprit objectif, à la fois commun et objectivé, dans les différents domaines de la vie publique: droit, art, religion, technique, mœurs, etc. Le mystère d'une modernité imparfaite est, d'un côté, de n'avoir plus de contextes institutionnels stables, et de l'autre, de ne pouvoir non plus se passer de toute institutionnalisation. Fluidification et condensation sociale se rencontrent dans un rythme d'action réciproque que la sociologie doit saisir en déterminant les limites spatio-temporelles de la légitimité des institutions ainsi que de la validité des assomptions de valeur. Simmel a exploré les différentes directions prises par l'activité humaine dans son processus d'objectivation, entraînant la constitution de mondes relativement autonomes. Ce mouvement général et les crises qui en résultent sont au centre de son analyse de la modernité, exprimée au mieux par sa Philosophie de l'argent. Puisque son propos n'avait rien d'économique, elle conserve toute sa pertinence diagnostique, ainsi que sa force de provocation ${ }^{2}$.

\section{UNE GUVRE À REDÉCOUVRIR}

L'effet d'une œuvre passe par sa relecture, qui seule permet la redécouverte, les surprises, que l'hommage habituel rend improbable. Alors que l'édition complète des écrits de Simmel touche à son terme (un volume d'index clôturera l'ensemble) ${ }^{3}$, le temps est donc venu pour une réappréciation. L'actualité de Simmel ne se dément pas depuis les années 1980 où la grande entreprise de la Gesamtausgabe a été mise sur pied sous la gouverne d'Otthein Rammstedt. L'effet de la publication des œuvres complètes a assurément renforcé sa perception comme un des grands classiques de la sociologie, en même temps qu'elle en a compliqué l'image traditionnelle. Ainsi, la réception italienne privilégiait l'essayiste et l'analyste de la vie culturelle, dans la proximité de Walter Benjamin et d'Ernst Bloch, alors que les pays anglo-saxons considéraient davantage le sociologue des villes, de l'argent et de la vie moderne, et que la réception française, de Raymond Aron à Raymond Boudon, s’intéressait en priorité au théoricien de l'histoire et au défenseur d'une certaine forme d'individualisme. Or ces lectures ne pouvaient différer tellement qu'en raison d'un état encore partiel des traductions, et plus profondément encore, en raison d'une méconnaissance de l'œuvre

2. Ce dont témoigne l'intérêt que lui porte André Orléan dans son projet de «refonder l'économie» à partir de l'autonomie de la valeur marchande fondée par la monnaie, comme Simmel l'avait vu, contre les fondations substantielles de la valeur sur le travail ou l'utilité, voir Orléan (2011).

3. Deux comptes rendus des volumes 18 et 21 saluent ici cette entreprise remarquable. 
simmelienne dans son ensemble. C'est bien le contexte spécifique de la genèse de l'œuvre qui se trouve singulièrement éclairé par les volumes 17, 22 et 23, parus de 2005 à 2008, qui rassemblent les petites contributions circonstancielles à des revues ainsi que les deux volumes de la correspondance. La possibilité d'un regard synoptique sur l'ensemble de cette production dans son temps, mais aussi dans sa pertinence pour les enjeux les plus actuels de la recherche, est ainsi maintenant offerte.

Peu à peu, la présence de la pensée de Simmel s'est affirmée dans le paysage francophone, comme l'indiquent les traductions, dorénavant nombreuses, les introductions à la sociologie (Martuccelli, 1999), l'inscription d'un extrait de la Philosophie de l'argent à un concours national en France en 2009 ou encore les récents numéros thématiques qui lui ont été consacrés, l'un par la revue Sociétés de Louvain en 2008 (coordonné par Patrick Watier), l'autre par la revue Emulations en janvier 2009 autour des thèmes «Environnement, conflits, mondialisation» (revue en ligne, numéro coordonné par Grégoire Lits). C’est dire que le temps pour une appréhension réfléchie de ce renouveau est venu.

\section{PRINCIPE DE CE NUMÉRO}

La confrontation avec l'œuvre de Simmel ne laisse pas de produire le plus souvent un certain embarras herméneutique. Elle ne se laisse reconduire ni au statut de standard de la formation sociologique à l'instar de Durkheim ou de Weber ni à celui d'un écrivain-philosophe, où on aurait aimé le ranger pendant la renaissance des études simmeliennes des années 1980. Par conséquent, le lecteur est confronté à un certain nombre d'idées reçues tant positives que négatives, qui favorisent des approches sélectives du corpus, en fonction des différents intérêts et des thèses socio-politiques qui les inspirent. Au-delà de l'aspect critique et diagnostique des tensions de la modernité, qui ont assuré son renom, l'apport constructif de Simmel pour l'auto-compréhension de la sociologie doit être mis en avant, car il est trop souvent minoré. Les propositions d'interprétation du présent numéro de Sociologie et sociétés entendent contribuer à la discussion en suggérant des voies nouvelles et d'autres perspectives. Elles naissent d'un débat sur l'actualité de Simmel qui s'est développé dans l'échange franco-allemand et qui a eu pour catalyseur le Centre Marc Bloch de Berlin ${ }^{4}$. Les quatre sections du volume (la quatrième réunissant un «dossier» qui illustre la vitalité des études simmeliennes et les apports que la recherche donne à son actualité) proposent ainsi un prisme thématique qui encadre la complexité de l'approche de l'œuvre de Simmel dans les débats courants sur le monde social. Elles se penchent d'abord sur la place de la sociologie simmelienne dans les dispositifs épistémologiques des sciences sociales (1. Interpréter la société) pour aborder ensuite la question du rendement de ses catégories pour l'analyse des émergences sociales contemporaines (2. Crises modernes). La lecture simmelienne de la modernité par le biais de la théorie de l'individualité qui fait

4. Situé à Berlin, à l'angle de la Friedrichstrasse et de la Leipzigerstrasse, dans un cadre familier de Simmel, le Centre Marc Bloch est un centre franco-allemand de recherches en sciences sociales. 
l'objet de la troisième section (3. Individualité). En clôture du numéro, des parcours moins connus de la réception de l'œuvre sont explorés à partir d'un dossier (4. Les actualités de Simmel) qui jette un regard sur l'édition et sur la littérature secondaire récente.

Ces quatre ensembles problématiques sont précédés d'une réflexion épistémologique sur le statut du discours scientifique simmelien (Denis Thouard). Le premier ensemble exprime l'apport original de l'œuvre de Simmel aux sciences sociales comme à la théorie de la société. C'est l'objet des réflexions sur le rôle de la quantification dans l'analyse sociologique (Zoreh Bayatrizi, Thomas Kemple), sur le statut de la science historique (Florence Hulak) et la signification du projet simmelien de théorisation de la société dans une perspective de théorie politique (Niccola Marcucci). Un second volet invite à une confrontation à trois cas particuliers de crises. Les avatars de la crise de la culture dans ses aspects contemporains, dans les domaines de l'information (Annette Disselkamp), de la finance (Alain Deneault) ou du multiculturalisme (Gregor Fitzi), permettent d'éprouver les ressources herméneutiques de la théorie simmelienne. Les crises ébranlent la stabilité des ordres, il en résulte un effet libérateur pour la figure de l'individu, qui peut revêtir des formes contrastées. C'est un des apports éminents de la sociologie simmelienne que d'avoir pensé l'individu moderne dans un cadre complexe, qui est ici mis en lumière dans sa cohérence (Hans-Peter Müller), et discuté de façon critique relativement à Max Weber et Durkheim (Catherine ColliotThélène, Jean Terrier). Enfin, un dernier ensemble évoque l'actualité permanente de l'œuvre simmelienne à travers sa réception italienne, précoce et nourrie (Claudia Portioli), l'appropriation qu'en fit Walter Benjamin présentée à partir un ouvrage récent (Léa Barbisan), enfin par un regard sur deux volumes de la GSG, l'un consacré aux écrits anglais qui permet de dessiner les contours de ses premiers lecteurs anglosaxons, l'autre à ses cours, d'après les notes de nombreux auditeurs, qui permet d'entrer dans sa pratique d'enseignement. La traduction d'un texte synthétique de 1916, «Transformation des formes culturelles», complète l'ensemble (Barbara Thériault).

\section{UN PARCOURS RÉFLEXIF}

Les textes qui suivent engagent une relecture, mais aussi une reprise libre de thèmes et de problèmes simmeliens. Il ne peut s'agir que de le lire comme lui-même aurait lu un auteur antérieur, sans dévotion pour la lettre, mais dans le souci de s'en instruire à nouveaux frais. Une réflexion herméneutique sur l'entrée dans l'univers de Simmel ouvre le volume. Contre l'image courante de l'observateur génial mais superficiel de la modernité, Denis Thouard dessine le contour d'un Simmel en penseur d'une science en train de se faire. Dans cette perspective et pour en finir avec l'image récurrente d'une "frivolité simmelienne», c'est avant tout la question de la scientificité de son œuvre qui doit être affrontée. Les ruptures simmeliennes avec les conventions académiques relèvent du caractère particulier de sa réflexion, qui se traduit par un rapport critique au langage ainsi que par une constante inspiration philosophique. L'accès à 
son travail théorique passe ainsi par une interrogation sur son style scientifique et, parlà, sur l'intention fondamentale de son œuvre. Les aspects incriminés de la prose de Simmel sont bien sûr à rapporter aux vicissitudes de sa carrière, à sa pratique du séminaire, à sa conception de la sociologie centrée sur les «formes de socialisation». Mais la nature de l'essai simmelien, notamment par contraste avec les conceptions d'Adorno qui faisait de l'écriture philosophique un enjeu esthétique, ne vise pas à faire œuvre. Il est une invite à la pensée, une provocation, plutôt qu'une écriture qui vaudrait pour elle-même. Simmel subordonne l'écriture à l'intention philosophique. Chez lui en effet, la forme est secondaire par rapport au sens de la question. Il s'agit de poser des problèmes plus que d'établir une doctrine. Elle est ainsi parfaitement adéquate au projet même de sa sociologie.

\section{INTERPRÉTER LA SOCIÉTÉ}

Interpréter la société suppose d'établir le rapport de la théorie sociologique avec les méthodes de la recherche empirique. La concurrence entre les méthodes quantitatives et qualitatives est au centre du débat épistémologique des sciences sociales contemporaines. La question du rapport des auteurs classiques avec la recherche quantitative s'impose ainsi à l'attention si on se penche sur la différence de leur approche par rapport à l'actuelle division du travail à l'intérieur de la sociologie. Zohreh Bayatrizi et Thomas Kemple examinent le rôle de la recherche empirique dans le travail des auteurs (canoniques ou non) de la sociologie au tournant des $\mathrm{XIX}^{\mathrm{e}}$ et $\mathrm{XX}^{\mathrm{e}}$ siècle. Étant le seul à formuler de façon explicite une théorie de la détermination quantitative des groupes sociaux, Simmel devient alors le point de départ de l'étude. Le rôle de l'analyse quantitative chez Durkheim et Tarde, Marx et Engels, ou Max et Alfred Weber fait l'objet d'une étude historique. Peut-on évaluer l'importance que la recherche statistique a eue pour établir les grandes théories des auteurs classiques? La réponse va être donnée dans le cadre d'un exposé qui montre que la particularité de l'approche classique tient à l'intégration de la théorie avec les données de la recherche empirique: c'est dans ce contexte que Simmel put jouer un rôle décisif et souvent méconnu.

La situation de la sociologie parmi les sciences sociales n'est pas seulement fonction de son rapport à la quantification des phénomènes sociaux, elle est aussi fonction de leur historicité. Dans la perspective de Florence Hulak, l'importance de la réflexion sur l'histoire dans l'œuvre de Simmel se comprend par le rapport de la sociologie à la science historique. L'histoire ne peut plus être définie comme science du passé, si elle participe à la connaissance du présent, notamment en mettant en évidence la façon dont celui-ci est travaillé par le passé. La question du statut épistémologique de l'intégration des sciences historique et sociale s'impose ainsi à l'attention. Tout au long de sa carrière, Simmel s'est efforcé de construire une épistémologie de la science historique, mais n'est jamais parvenu à en proposer une conception véritablement unifiée. L'ayant d'abord conçue comme premier niveau de la connaissance en sciences humaines, préparant le travail sociologique, puis comme science des formes individuelles, distinctes des formes sociologiques, il semble l'avoir toujours pensée dans le cadre 
d'une interrogation sur la représentation du passé. Les prémisses d'une autre conception de l'histoire peuvent toutefois être décelées dans l'œuvre de Simmel. Elles permettent de complexifier l'image sociologique d'une société, en reliant l'articulation de la logique interne des actions réciproques à celle des héritages du passé.

La réflexion politique semble ne jouer qu'un rôle mineur dans l'œuvre de Simmel. Si l'on se penche sur les transformations de la question classique de l'obligation dans la pensée du XIx ${ }^{\mathrm{e}}$ siècle, sa présence devient toutefois plus importante qu'on ne le penserait de prime abord. C'est la thèse que développe Nicola Marcucci. À l'époque moderne, la conception du rapport entre individu et société prend la forme d'une réflexion sur l'obligation politique. Constatant des tensions entre les acteurs et la structure sociale issue de l'émergence du conflit social moderne, la sociologie prend acte de la crise de cette formule. Les doctrines du progrès ne suffisent plus à intégrer les perspectives individuelles et collectives. La deuxième génération des sociologues réinvestit la problématique de l'obligation d'une perspective originale. Une nouvelle conception de l'individu se dessine: l'agencement entre la loi et l'autonomie devient problématique en raison de la «déterritorialisation» de l'universalisme de la loi au profit de sa validité régionale. Une nouvelle conception de la loi à fondement sociologique est donc nécessaire. Le propos de Simmel se fonde sur la critique de la morale kantienne et élabore une synthèse positive entre l'individualité et l'égalité pour repenser l'obligation des modernes. La «loi individuelle» constitue ainsi le tiers qui permet de dépasser l'ambivalence moderne entre loi et individualité tout en se détournant de l'ascèse politico-méthodologique de Weber.

\section{CRISES MODERNES}

Avec la progression de la complexité sociale, la théorie sociologique rencontre de nouveaux terrains de recherche qui défient ses capacités d'explications. C'est entre autres le cas des crises d'intégration des sociétés contemporaines qui provoquent le débat sur le multiculturalisme. Le phénomène de la crise de la culture se trouve ainsi enrichi d'une nouvelle dimension. Dans quelle mesure une société avec un haut niveau d'hétérogénéité culturelle peut-elle accomplir son intégration? Dans ce contexte transformé par l'universalisation de la Wechselwirkung, il s'agit alors de vérifier jusqu'à quel point la théorie simmelienne de la culture contribue à saisir les enjeux de cette nouvelle émergence sociale. C'est le propos de Gregor Fitzi. Suivant l'analyse de Will Kymlicka, il faut dépasser d'un côté les mythologies du melting-pot et de l'ethnicmosaic et de l'autre le concept monodimensionnel de multiculturalisme pour introduire la distinction entre «multi-nationalité» et "poly-ethnicité». Une clarification du concept de culture et de son rôle pour l'intégration des sociétés complexes devient alors une condition préalable pour la compréhension du phénomène. La théorie de la culture de Simmel dépasse la dichotomie marxienne entre base et superstructure et la rapporte à une conception de la société à différenciation fonctionnelle. Elle permet ainsi de voir dans la culture un moteur d'intégration socio-structurelle. Les tensions dues à la crise de la culture se laissent par conséquent lire comme les indicateurs des 
dysfonctionnements structurels des sociétés complexes. Appliquer cette approche à l'analyse du multiculturalisme signifie déconstruire le réductionnisme ethnique qui en interprète la problématique en termes de clash of civilisations. La tension entre identités culturelles est à interpréter comme l'expression d'une crise de simplification culturelle qui amène les communautés (autochtones ou non) à rechercher leur salut dans l'immunisation des rapports sociaux. La particularité du phénomène réside dans le fait que cette négation de l'horizon de référence de la fonction socio-structurelle de la culture intéresse aussi les efforts d'intégration les plus avancés. Le risque est alors de s'inscrire dans une nouvelle mythologie de l'intégration: le «multi-communautarisme». Pour y échapper une évaluation de la fonction socio-structurelle de la culture dans les sociétés complexes s'impose, qui intègre l'analyse sociologique de Simmel et l'anthropologie philosophique de Helmuth Plessner sur le fond de la conception du multiculturalisme de Charles Taylor.

Le secret n'est généralement pas considéré comme un paradigme de référence pour l'étude de la connaissance interpersonnelle. Rétablir le secret dans ses droits, c'est l'opération intellectuelle du chapitre homonyme de la Sociologie de Simmel qui, selon Annette Disselkamp, prend l'Aufklärung à contrepied. Il n'y a plus d'impératif du dévoilement. Le secret est fonctionnellement lié à la constitution du social et recouvre ainsi une nouvelle légitimité. Le regard se penche sur le mensonge et sur la limitation de l'accès à l'information comme techniques de la vie sociale: ce sont des constituants de la présentation de soi dans la mise en scène théâtrale qui fonde l'interaction. Simmel met ainsi en évidence l'arrière-plan anthropologique de l'association qui s'entrecroise avec la logique de la différenciation des sphères fonctionnelles. Renforcée par l'échange monétaire fondé sur les contacts ponctuels et la confiance sans familiarité, la limitation de la connaissance réciproque devient alors la norme dans la société moderne. Elle se traduit par des identités multiples, l'instauration de distances, la connaissance fragmentaire ou encore le secret comme protection. L'actualité de la théorie simmelienne se montre avant tout dans l'analyse de la société numérique, dont les réseaux sociaux se laissent lire comme transposition technologique de la problématique du secret épistolaire. Pour accomplir la compréhension sociologique ne faut-il pourtant pas sacrifier le paradigme épistémologique du secret? Pas nécessairement. La notion de discrétion se retrouve en effet à la base de la compréhension réciproque des êtres humains dans le respect de leur nature qui prévoit la tutelle d'une sphère intime non accessible. Cela amène à conclure sur le caractère circulaire du lien entre la connaissance et la compréhension, qui se fonde sur la notion d'une pénétration de la réalité à l'appui et dans le respect du secret.

Si la Philosophie de l'argent peut encore nous parler plus d'un siècle après sa parution, c'est d'abord en vertu de son questionnement original qui, prenant du champ par rapport aux différentes doctrines économiques de son temps, n'en a pas partagé la péremption. Simmel s'est interrogé, avec une sorte de naïveté philosophique composée, sur les catégories fondamentales impliquées dans l'opération économique. La valorisation et l'institution de la valeur, la logique de l'échange sont reconstituées dans 
l'effort d'en fournir une plus grande lisibilité. Mais c'est le croisement de cette logique propre des échanges et de la logique des actions des acteurs, aux effets parfois diamétralement contraires aux attentes, qui institue la complexité sociale et un défi à l'intelligibilité globale de tels processus. Alain Deneault porte son regard précisément sur ces logiques de la perversion économique dont Simmel, au troisième chapitre de son œuvre, a livré une tératologie très complète. L'introduction des séries téléologiques et de leurs retournements complexifie radicalement la situation. Quand les moyens sont pris pour des fins, la perversion inévitable et généralisée s’installe. Simmel en brosse un tableau savoureux, de l'avare au dispendieux, dont Deneault montre qu'il n'a rien perdu de son potentiel critique. Il en fait, d'après Deleuze, autant de "personnages conceptuels » qui illustrent, aujourd'hui comme naguère, les pathologies sociales de l'argent, permettant de décrypter par avance les excès de la finance actuelle. Allant plus loin que Simmel, Deneault dégage une véritable «philosophie de la perversion » des analyses de l'effet pervers, qui débouche sur la peinture d'une situation psychique inquiétante, voire délabrée, qui traverse la culture contemporaine. La logique de la perversion tend à se substituer à celle de la réciprocité, mettant la société en péril d'elle-même.

\section{INDIVIDUALITÉ}

Comme le veut la vulgate sociologique, Simmel est le classique inconnu, inactuel et sans héritiers. Ce qui frappe dans ces conditions est le fait que plusieurs aspects de sa théorie font néanmoins partie du mainstream de la discipline ${ }^{5}$. Selon Hans-Peter Müller, il convient alors de se demander s'il en va de même pour son «individualisme». Dans sa réflexion, il y a un lien théorique indissoluble entre le questionnement sur «comment la société est-elle possible» et celui de l'individualité. Les deux se fondent sur un hiatus entre possibilité et réalisation. L'approche de Simmel est à la fois philosophique et sociologique, au sens où chez lui l'analyse anthropologique et éthique de l'individualité encadre l'analyse structurelle et culturelle. Dans la perspective anthropologique, l'être humain est vu comme «être de la limite». Un animal qui est tenu lui-même de fixer ses propres limites pour les dépasser en permanence. Ainsi se développe une théorie de l'homo duplex qui se relie à celle des a priori de la sociologie. La contemporanéité de l'être social et de l'être par ailleurs se traduit dans celle de l'être de la limite qui a un pied dans la société et un pied à l'extérieur. La réflexion sur l'individualité se relie ainsi à l'analyse de l'élément structurel de l'association qui fait la particularité de la théorie simmelienne de la différenciation sociale. Cette dernière a un potentiel libérateur qui se trouve pourtant en décalage avec le développement culturel. C'est ainsi qu'une théorie de la culture comme «besoin d'individualité» prend place au centre de la conception simmelienne de la modernité. La crise de l'individualité moderne ne connaît pas de solution toute faite. Seule une minorité des

5. Voir l'anthologie de textes de Simmel éditée récemment par O. Rammstedt (2008) sous le titre Individualismus der modernen Zeit. 
tentatives d'individualisation réussit véritablement. Simmel préfigure ainsi une critique de la société de consommation qui propose aux masses l'ersatz d'une individualité aboutie, une simple «individualité light» en somme. La tragédie de l'individualité pourrait-elle pourtant être dépassée par une éthique individuelle comme celle que préfigure le dernier Simmel avec l'idée de «loi individuelle » ? La réponse est à chercher dans les études sur les individualités accomplies, en particulier dans le Goethe. L'actualité de la théorie simmelienne de l'individualité est donc fournie par ce double niveau argumentatif qui ne se contente pas de prendre acte d'un individualisme à large échelle mais vide, comme le fait la sociologie d'aujourd'hui.

L'encadrement de la liberté moderne présuppose une conception critique des liens entre l'individu et la société. Une assomption qui vaut d'autant plus pour une comparaison des théories sociologiques de l'individualité chez Simmel et Weber. Pour comprendre les différences entre leurs positions, il est toutefois nécessaire d'adopter une perspective interculturelle franco-allemande. C'est la démarche de Catherine Colliot-Thélène. Si selon Durkheim la modernité comporte une substantialisation de la personnalité individuelle, pour Simmel elle se caractérise par sa dépersonnalisation et son caractère anonyme. Il y a chez les auteurs allemands une sensibilité particulière pour l'impersonnalité, vue comme dimension critique de l'altérité anonyme, alors que, sous l'influence de Comte, le souci pour la cohésion sociale s'installe au centre de la réflexion de Durkheim. Les sociologues allemands sont plus sensibles quant à eux au problème de l'unité et de l'autonomie de l'individu. Sur ce point, la position de Simmel est très radicale. Se limitant au constat du changement des liens d'obligation véhiculés par l'argent, il refuse tout résidu de nostalgie de la communauté qu'on peut constater encore chez Durkheim ou Tönnies et accepte les formes modernes de la vie collective. Si le républicanisme de Durkheim se préoccupe de limiter les effets de décomposition dus à l'individualisme surtout sur le plan économique, pour Simmel celui-ci est un acquis de la modernité. Il ne faut pourtant pas penser que Simmel ait une attitude non critique vis-à-vis du libéralisme économique. Avec celui-ci, en effet, l'individualité personnelle risque d'être abolie au profit de la somme des rôles sociaux qu'elle exerce. Ce diagnostic recoupe les analyses de Weber. Comment sauver l'héritage des droits de l'homme dans un contexte où les liens sociaux sont complètement mécanisés? Simmel croit pouvoir aller au fond de la question. En termes d'histoire de la culture, il s'agit d'envisager une nouvelle synthèse de Kant et Goethe qui tienne compte de Nietzsche. Au fondement de son projet de synthèse entre les individualités quantitative et qualitative, on retrouve pourtant l'idée de personnalité, violemment critiquée par Weber. À la différence de Simmel, ce dernier met le Goethe des Wanderjahre au centre de l'identité moderne: la renonciation à l'idéal de l'autoréalisation à tout prix, l'ascétisme du mode de vie bourgeois, la division du travail. Est-ce une critique du Simmel goethéen? Oui et non. C'est plutôt l'absence d'une théorie de la domination qui constitue le problème de la théorie simmelienne de la loi individuelle, en l'empêchant de replacer la lutte de l'individualité moderne pour son autonomie dans le cadre d'une analyse de l'inégalité sociale. 
La comparaison des doctrines de Durkheim et Simmel sur l'individualité engage enfin une analyse du rapport entre les dimensions singulière et collective de ce concept. Peut-on, en sociologie, transférer l'idée d'individualité des personnes naturelles aux personnes collectives? Jean Terrier propose une perspective comparative pour répondre à la question. Le défi théorique auquel Simmel et Durkheim se trouvent confrontés est d'échapper aux nombreux théorèmes substantialistes typiques des théories sociales de la deuxième partie du $\mathrm{XIX}^{\mathrm{e}}$ siècle. Pour Durkheim la solution réside dans la fondation de la sociologie sur une théorie des représentations collectives. Si le développement de l'individualité moderne met en danger la cohésion sociale, alors il faut repérer les moyens pour renforcer et actualiser les représentations collectives intériorisées pendant la socialisation. Il y a alors un lieu de coagulation de la conscience collective qui garantit la cohésion sociale en sorte que, face aux crises sociales, les idéaux collectifs se régénèrent. C'est la théorie de l'effervescence collective que Durkheim développe sur le fond des batailles politiques de la troisième République. Simmel par contre est porteur d'un antiréalisme radical. Il recourt à la théorie de la différenciation sociale pour surmonter tant le collectivisme que l'individualisme dogmatique. Les processus sociaux d'objectivation et de subjectivation se développent parallèlement sans être en mesure de fonder une théorie unitaire mais seulement une théorie conflictuelle de la personnalité. Sa lutte contre les tendances qui la dispersent est alors aussi une lutte contre les représentations collectives. On est ainsi confronté à deux conceptions différentes de la crise de la modernité. Durkheim insiste sur la nécessité de réaffirmer la vie collective; Simmel sur celle d'un assouplissement du social qui risque d'étrangler l'individu. Si l'on considère la complexité de leur réflexion, la divergence entre un «Durkheim holiste» et un «Simmel individualiste» n'est toutefois pas à prendre à la lettre. Tous les deux sont en même temps des penseurs de la différenciation sociale et de l'individualité. Ils diffèrent essentiellement dans la considération de l'ascendant moral et de l'autorité sur les comportements sociaux. De là découle aussi leur perception discordante de la distinction terminologique entre personnalité et individualité collective.

\section{LES ACTUALITÉS DE SIM MEL}

Les modalités de la réception de l'œuvre de Simmel au $\mathrm{xx}^{\mathrm{e}}$ siècle sont une des clés de la compréhension de sa présence-absence paradoxale dans le paysage des sciences sociales. Elles rendent compte de la portée de sa redécouverte contemporaine. Le dossier qui lui est consacré permet de s'en rendre compte.

D'une façon qui transcende les disciplines et semble faire l'objet d'un consensus de plus en plus large, l'œuvre critique et théorique de Walter Benjamin s'est installée au rang des références omniprésentes de notre réflexion. Souvent associé aux «sciences de la culture», son rayonnement va bien au-delà. Il était donc particulièrement intéressant de se demander ce qui pouvait constituer l'attrait si affirmé de cette œuvre, qui n'est pas sans présenter des affinités avec celle de Simmel. D'une part, bien que discrètement, Benjamin a connu celle-ci et s'y réfère à l'occasion, notamment dans son 
projet des Passages. D'autre part, le caractère transdisciplinaire et souvent anarchiste de ses questionnements a inauguré une voie d'approche qui fut exploitée par Benjamin, lequel confronta ce questionnement aux archives. Prenant appui sur la publication récente de la monographie de Marian Mičko (2010) consacrée aux deux auteurs, Léa Barbisan permet de faire le point sur ce dossier, caractéristique de la réception de Simmel, à la fois inspirateur décisif et régulièrement oublié, voire rejeté. Pour une part significative de son œuvre d'analyste culturel, il apparaît ainsi que celle de Benjamin l'a prolongée, approfondie, radicalisée mais aussi recouverte.

Si l'on considère maintenant un exemple de réception à l'échelle d'un pays, le cas de l'Italie livre également des enseignements inattendus. La réception italienne des œuvres de Simmel a connu un développement qui la distingue des toutes les autres. Comme le montre Claudia Portioli, elle a été continuelle et s'est concentrée au début surtout sur la philosophie de Simmel. Ceci tient en grande partie au fait que plusieurs philosophes italiens suivirent lors de leurs études à Berlin les cours de Simmel. De retour en Italie, certains se sont engagés dans la traduction de ses traités de philosophie comme les Hauptprobleme der Philosophie (1919) et la Lebensanschauung (1938) ainsi que d'une série d'essais. La première réception est donc importante, mais aussi caractérisée par une certaine stylisation de Simmel en champion du relativisme moral. La lecture de Banfi fait cependant exception, puisqu'il arrive à saisir positivement la potentialité analytique de la pensée de Simmel en tant que "philosophie de la crise». En revanche, la réception de Simmel par la philosophie officielle de Croce et de Gentile est demeurée très superficielle. Ce sont plutôt les outsiders comme Caffi, un intellectuel libertaire et révolutionnaire, qui aiment Simmel. Le début de la réception sociologique de Simmel en Italie se situe en revanche au passage des années 1970 aux années 1980 et est lié à la crise du marxisme. Après il y a pourtant eu un développement explosif des études et de l'activité de traduction. Cette dernière est certainement une des caractéristiques majeures de l'approche italienne d'un auteur sans école de référence comme Simmel.

Le volume des écrits parus en anglais (GSG 18) complète celui qui contenait les textes en français et en italien (GSG 19). Son évocation permet d'aborder concrètement les conditions de sa première lecture, notamment outre-Atlantique. De même, le volume réunissant les notes de cours de Simmel et de ses auditeurs (GSG 21) permet une incursion dans son amphithéâtre et suggère le décalage entre l'écrivain et le professeur. La considération de la diversité de son auditoire, toujours nombreux à se presser à ses cours, est une indication de première importance pour apprécier le mode d'influence de cette pensée. L'adjonction de la traduction d'un texte relativement tardif de la production de Simmel permet d'apprécier les modes de condensation de ses idées fondamentales sur la culture, soulignant la continuité de sa pensée dans son renouvellement. L'évocation des toutes dernières manifestations culturelles, comme ici avec le futurisme, montre un sociologue attentif aux signes de son époque, et soucieux, par son propre effort théorique, d'en indiquer les crises. 
Les formes culturelles se transforment comme les formes sociales, avec un rythme peut-être plus rapide. Simmel s'est constamment efforcé d'en suivre et d'en comprendre les dynamismes. Il a élaboré une réflexion philosophique autant que sociologique pour penser son temps, sans jamais chercher à réduire les phénomènes à ses hypothèses interprétatives. La conviction du caractère décisif des réciprocités sociales l'aura inspiré dans cette mise à jour perpétuelle. Plusieurs des études ici réunies indiquent précisément quel peut être l'apport des conceptions de Simmel pour le déchiffrement de notre monde en mutation. Il serait en effet vain, compte tenu de l'intention fondamentale de cette pensée, d'en proposer une approche visant à la figer dans une théorie aboutie, dans la figure d'un classique tutélaire de la discipline, voire d'une référence à la mode. Les différentes tentatives de contextualisation présentées ici visent au contraire à revenir au noyau original de son œuvre, pour en sonder les potentialités et les forces. C'est l'historisation qui permet de se réapproprier une œuvre: la confrontation avec ses pairs, de Durkheim à Weber, aide à la retrouver. C'est aussi pourquoi, l'édition complète ayant remis en circulation la totalité de ses textes, il convient de se les approprier pour y réagir et les penser à nouveaux frais. Nous espérons que ce numéro de Sociologie et sociétés y contribuera, illustrant à sa façon l'interdépendance entre la lecture des textes et l'intelligence du présent.

\section{BIBLIOGRAPHIE}

Martuccelli, D. (1999), Sociologies de la modernité, Paris, Gallimard.

OrléAN, A. (2011), L'empire de la valeur. Refonder l'économie, Paris, Seuil. 